\title{
Editorial: Diversity and Evolution of Animal Venoms: Neglected Targets, Ecological Interactions, Future Perspectives
}

\author{
Maria Vittoria Modica ${ }^{1,2 *}$, Kartik Sunagar ${ }^{3}$, Mandë Holford $^{4,5,6,7}$ and Sébastien Dutertre ${ }^{2}$ \\ ${ }^{1}$ Department of Biology and Evolution of Marine Organisms, Stazione Zoologica Anton Dohrn, Naples, Italy, ${ }^{2}$ Institut des \\ Biomolécules Max Mousseron (IBMM), UMR 5247, CNRS, Université de Montpellier, Montpellier, France, ${ }^{3}$ Evolutionary \\ Venomics Lab, Centre for Ecological Sciences, Indian Institute of Science, Bangalore, India, ${ }^{4}$ Department of Chemistry and \\ Biochemistry, Hunter College, New York, NY, United States, ${ }^{5}$ PhD Programs in Biochemistry, Chemistry, and Biology, The \\ Graduate Center of the City University of New York (CUNY), New York, NY, United States, ${ }^{6}$ Department of Invertebrate \\ Zoology, The American Museum of Natural History, New York, NY, United States, ${ }^{7}$ Department of Biochemistry, Weill Cornell \\ Medicine, New York, NY, United States
}

Keywords: venom, evolutionary ecology, biodiversity, comparative genomics, toxins, biotechnology

\section{OPEN ACCESS}

Edited and reviewed by:

Li Chen,

Institute of Zoology (CAS), China

${ }^{*}$ Correspondence:

Maria Vittoria Modica

mariavittoria.modica@szn.it

Specialty section:

This article was submitted to

Chemical Ecology,

a section of the journal

Frontiers in Ecology and Evolution

Received: 17 January 2020 Accepted: 02 March 2020

Published: 24 March 2020

Citation:

Modica MV, Sunagar K, Holford M and Dutertre S (2020) Editorial:

Diversity and Evolution of Animal Venoms: Neglected Targets, Ecological Interactions, Future

Perspectives. Front. Ecol. Evol. 8:65. doi: 10.3389/fevo.2020.00065

\section{Editorial on the Research Topic}

Diversity and Evolution of Animal Venoms: Neglected Targets, Ecological Interactions, Future Perspectives

Many animals rely on the production of complex venoms for predation and defense. Their venom arsenal is often a mix of bioactive compounds, produced in specialized tissues and delivered through a broad range of fascinating anatomical structures. Venoms have evolved through millions of years of natural selection, in intricate co-evolutionary "arms races," where the venomous animal plays the prey, the predator, or often both. As a result, venoms are extremely potent, being usually effective at a very low concentration via highly specific interactions with key physiological targets. Many venom toxins target the neuromuscular system, while others possess extremely potent anticoagulant, anesthetic, and hypotensive activities, making them important biotechnological tools. This Research Topic will discuss the diversity and evolution of venom with a focus on expanding beyond model systems to examine neglected taxa, and characterizing ecological interactions in venomous organisms.

The biotechnological relevance of venom compounds has been relatively well exploited in snakes, from which captopril ${ }^{\circledR}$, one of the first FDA approved drugs derived from animal venoms, was obtained in the 1970s. Captopril is a potent inhibitor of the angiotensin-converting enzyme (ACE) used to treat hypertension and heart failure. However, as reviewed in this Research Topic, potential exploitation of snake venom as a bioresource goes well beyond biomedicine, including applications in diagnostics and in cosmetics (Ferraz et al.).

In addition to having an indisputable potential in drug discovery research, venomous taxa are also highly suitable for investigating ecological interactions between organisms, along with their evolutionary implications. Venom composition is highly variable, both among and within species, as it is shaped by various biotic and abiotic factors, including prey and predator pressures, environmental conditions, and phylogenetic histories. Despite the technological advancements in the last two decades, especially in the areas of high-throughput sequencing technologies, mass spectrometry and genomic manipulations, the precise role of the aforementioned factors in shaping the animal venom arsenal is yet to be fully elucidated. 
In this Research Topic, the importance of broadening investigation to neglected taxa and concepts has been emphasized by Jackson et al. who point out that overlooked lineages can be of great interest for toxinologists, especially in the context of venom ecology and evolution. Their review highlights that, among snakes, only the species that are capable of inflicting clinically severe envenomations in humans have been the focus of research, while most "non-front fanged" species have been largely neglected despite their high phylogenetic and ecological diversity (Jackson et al.).

The link between trophic diversification and venom evolution makes the investigation of neglected lineages with specific preys particularly promising in terms of novel activities, as here reviewed by Modahl and Mackessy. Recent studies have not only allowed the characterization of novel protein families in rear-fanged snakes (e.g., veficolins, matrix metalloproteinases, and acid lipases), but have also led to the identification of new functions in well-characterized toxin families, including threefingers toxins (Modahl and Mackessy).

Similar to snakes, despite a long history of venom studies in spiders, a large number of taxa remain overlooked. In this Research Topic, Zobel-Thropp et al. describe, for the first time, the venom composition of the Pholcid spiders, popularly known as daddy long-legs, a diverse and ancient lineage of spiders that are generalist foragers of arthropods, including other spider species. Their work reveals the complexity of Physocyclus mexicanus venom, suggesting a primary role in its activity for the neprylisin family, and highlighting its potent toxicity in arthropods with negligible effects on humans (Zobel-Thropp et al.).

Among cnidarians, corals represent yet another group of venomous organisms that are worthy of further investigation. However, to date, coral venom characterization has been hampered by the inherent technical difficulties. As pointed out in this Research Topic, even the best-characterized coral toxins, the SCRiPS, have not been subjected to rigorous biochemical and functional testing, despite exhibiting potent neurotoxic and antimicrobial activities (Schmidt et al.).

Beside the focus on untapped taxa, several contributions to this Research Topic tackle the origin of venom complexity at different levels. At the intraspecific scale, population proteomics combined with multivariate statistical analyses allowed the detection of signatures of natural selection in two species of parasitoid wasps, and the identification of populationspecific proteins, some of which are responsible for virulence (Mathé-Hubert et al.). This work reveals the eco-evolutionary feedback between the organisms and the ecosystems, with important implications for biological control.

In scorpions, long-scale evolutionary adaptations to different environments have been demonstrated to affect both venom composition and stinger morphology. Here, Evans et al. highlight the role of individual-level adaptations in the optimization of the balance cost-benefit of venom use. These adaptations include both behavioral plasticity in response to predator/prey identity, and modification of venom composition according to different predatory pressures (Evans et al.).

The molecular mechanisms underlying venom complexity are still not fully understood, but a dominant role was attributed to gene duplication events. To comprehend the origin and evolution of toxin genes and morphological adaptation, a comparative genomic approach may be crucial. Comparative genomics revealed the importance of mechanisms that do not fit the classical model of gene function evolution, such as the cis-regulated changes in the venom gland expression in the parasitoid wasp, Nasonia vitripennis, and the horizontal transfer of toxin genes from bacteria to the cnidarian Nematostella vectensis (Drukewitz and von Reumont).

Similarly, by the integration of transcriptomic, proteomic and genomic data of the common house spider, Parasteatoda tepidariorum (Haney et al.), shed light on the role of alternative splicing in generating venom complexity. This process may be influenced by environmental cues, representing a possible mechanistic link between environmental pressures and adaptive changes in venom composition. Thus, in absence of preceding gene duplication events, alternative mechanisms may operate to modify venom composition (Haney et al.; Drukewitz and von Reumont).

Altogether, this Research Topic covers many of the leadingedge trends in venom research. These trends show great promise for advancing our knowledge about the enormous biodiversity on the planet, while identifying new toxins with diverse biotechnological potential.

\section{AUTHOR CONTRIBUTIONS}

MM wrote the original draft. SD, KS, and $\mathrm{MH}$ reviewed and edited the manuscript. All the authors read and approved the final manuscript.

\section{ACKNOWLEDGMENTS}

MM acknowledges funding from the European Union's Horizon 2020 research and innovation programme under grant agreement No 748902. MH acknowledges funding from Hunter College Presidential Faculty Scholar Fellowship.

Conflict of Interest: The authors declare that the research was conducted in the absence of any commercial or financial relationships that could be construed as a potential conflict of interest.

Copyright (c) 2020 Modica, Sunagar, Holford and Dutertre. This is an open-access article distributed under the terms of the Creative Commons Attribution License (CC BY). The use, distribution or reproduction in other forums is permitted, provided the original author(s) and the copyright owner(s) are credited and that the original publication in this journal is cited, in accordance with accepted academic practice. No use, distribution or reproduction is permitted which does not comply with these terms. 